\title{
Cómo enseñan gramática española los futuros académicos de la lengua: una experiencia docente en el Grado en Filología Hispánica
}

\section{How future Spanish language scholars teach grammar: a teaching experience in the Degree in Hispanic Philology}

ANA MANCERA RUEDA

ORCID https://orcid.org/0000-0001-5343-4935

Universidad de Sevilla

Departamento de Lengua Española, Lingüística y Teoría de la Literatura anamancera@us.es

Fecha de recepción: 20-11-2019

Fecha de aceptación: 24-11-2019

DOI: http://dx.doi.org/10.12795/9788447221912.103

Pp.: 2311-2336 


\section{Resumen}

Durante el primer cuatrimestre del curso 2019/2020 se ha realizado un ciclo de mejora de asignatura completa (CIMA) con los alumnos matriculados en Gramática española, materia que se imparte en el último año del Grado en Filología Hispánica. Siguiendo las directrices didácticas de la Red de Formación e Innovación Docente (REFID), la innovación se ha centrado en dos aspectos centrales: el desarrollo de clases impartidas por los propios alumnos y la evaluación mediante porfolio.

Palabras clave: Gramática Española, Grado en Filología Hispánica, docencia universitaria, experimentación docente universitaria

\section{Abstract}

During the first semester of the 2019/2020 academic year, a Improvement Cycle in Classroom (ICIC) has been carried out with the students enrolled in Spanish Grammar, a subject taught in the last year of the Degree in Hispanic Philology. Following the didactic guidelines of the Teacher Training and Innovation Network (REFID), innovation has focused on two central aspects: the development of classes taught by the students themselves and the evaluation through a portfolio.

Keywords: Spanish Grammar, Degree in Hispanic Philology, university teaching, university teaching experimentation

Jornadas de Formación e Innovación Docente del Profesorado | № 2 (2019) Esta obra se distribuye con la licencia Creative Commons 


\section{Introducción}

El título de este trabajo, de carácter humorístico, guarda relación con una actividad que planteé a los alumnos de la asignatura Gramática española el primer día de clase. Esta consistía en proponerles un viaje al futuro y, tras mostrarles una fotografia del Dr. Emmett Lathrop Brown -uno de los protagonistas de la trilogía Regreso al futuro, más conocido con el apodo de Doc-, les pedí que imaginasen cómo serían dentro de unas décadas. A continuación, proyecté una imagen de los miembros de la Real Academia de la Lengua Española y les insté a "representar el papel de académicos a los que se les ha encargado la redacción de la Más nueva gramática de la lengua española, jla definitiva!" -para ellos saltaba a la vista el carácter irónico de esta referencia intertextual a la Nueva gramática de la lengua española, publicada en 2009, y que constituye la obra fundamental en la que se basa el estudio de dicha asignatura-. Con este fin, dividí la clase en grupos de seis integrantes y les animé a ponerse de acuerdo sobre cómo debía ser la gramática que deseaban elaborar, para poner después sus ideas en común con el resto de los compañeros.

Dicha tarea, además de funcionar como "actividad de rompehielos", me permitió identificar los conocimientos previos de mis estudiantes acerca de esta rama de la lingüística. Por ejemplo, me di cuenta de que algunos de ellos confundían los contenidos propios de las gramáticas con los que habitualmente proporcionan los diccionarios o los repertorios de normas ortográficas. Además, muchos eran partidarios de elaborar una gramática descriptiva, pero no prescriptiva, lo que suscitó un gran debate en clase.

Por otra parte, me sorprendió de manera grata el hecho de que cuestionaran el enunciado de la actividad, en el que se les instaba a elaborar la gramática "definitiva" - una aserción que yo había formulado con un mero fin lúdico, 
pero que ellos interpretaron de manera literal-, ante lo que replicaron muy sabiamente que la lengua siempre estará sujeta a cambios, de ahí la imposibilidad de diseñar un tratado con la pretensión de que sirviera como referencia con carácter indefinido. Lo que no me sorprendió tan gratamente fue la propuesta de los integrantes de uno de los equipos de elaborar una gramática para niños de seis años ya que, al igual que Pons (2018), soy de la opinión de que transmitir el metalenguaje propio de la lingüística "en edades cortas roba tiempo para lo fundamental: aprender a expresarse, a leer con gusto, a saber hablar en público", máxime tratándose de escolares que prácticamente acaban de aprender a leer y a escribir.

En general, todos estaban de acuerdo con la necesidad de configurar su obra tomando como referencia un corpus de documentos representativos tanto de las variedades peninsulares como del español de América -en este punto reconocieron que habían estudiado algunos de los contenidos de la Nueva Gramática de la Lengua Española en la asignatura El español de América pero, al parecer, apenas se la nombraba en otras asignaturas-. Asimismo, casi todos los grupos hicieron propuestas en las que abogaban por el uso de las nuevas tecnologías, por ejemplo, para permitir que la consulta de su obra se realizara directamente a través del motor de búsqueda Google, para el empleo de mecanismos de reconocimiento de voces a la hora de plantear una consulta, y también proporcionando vídeos ejemplificadores de distintos fenómenos lingüísticos en la página web de la Real Academia Española, incluyendo en sus bases de datos ejemplos extraídos de las redes sociales e, incluso, uno de los equipos centró su exposición en la elaboración de la primera Gramática Millennial. Todo esto -al igual que el cuestionario inicial, del que hablaré más adelante- me permitieron forjarme una idea inicial de los conocimientos de mis alumnos. 


\section{Diseño previo del CIMA}

Para la elaboración del diseño previo de este "ciclo de mejora de asignatura completa" (Porlán, 2016), decidí basarme en los dos CIMA que he desarrollado previamente y en mi propia experiencia como docente de Gramática española, una materia que he venido impartiendo desde el curso 2004/2005 -si bien no de forma consecutiva-. El primer CIMA lo implementé en la asignatura Análisis del discurso y pragmática del español durante el curso 2015/2016, siguiendo las sabias enseñanzas de Eduardo García y Ana Rivero en el Seminario de profesores universitarios noveles de iniciación. Desde entonces, trato de aplicar lo allí aprendido a todas mis clases. En las III Jornadas de Docencia Universitaria, coordinadas por Rafael Porlán y Elisa Navarro, he contado mi experiencia (Mancera, 2016). El segundo fue un ciclo de mejora completo que desarrollé en la asignatura Análisis lingüístico de texto del español actual durante el segundo cuatrimestre del curso 2017/2018, en el marco de la Red de Formación e Innovación Docente del Profesorado de la Universidad de Sevilla (REFID), recogido en Mancera (2018). Mi innovación en este último CIMA consistió en dos aspectos: 1) el desarrollo de clases impartidas por los propios alumnos; 2) la evaluación mediante porfolio. Metodologías docentes en las que se basa también el presente CIMA.

Tras mi experiencia como docente de Gramática española a lo largo de varios cursos, he podido constatar que la mayoría de los estudiantes que acceden al cuarto curso de los estudios del Grado de Filología Hispánica manifiestan su predilección por las asignaturas que abordan aspectos vinculados con la literatura y muchos de ellos sienten, incluso, un rechazo considerable hacia algunas de las materias correspondientes al área de Lengua Española, después de haber cursado en años anteriores asignaturas con elevados índices de suspensos, como Gramática histórica del español, Historia del español I o Historia del español II. 
Por otra parte, los contenidos del programa de Gramática española suelen resultar de gran dificultad para los alumnos. No obstante, muchos de ellos se limitan a tomar apuntes sobre lo que va diciendo el profesor, sin preocuparse por entender lo que este explica. Con frecuencia, tales apuntes resultan incompletos y están plagados de incongruencias, ya que los estudiantes se muestran más preocupados por copiar todo lo que se expone en una clase magistral, que por seguir los razonamientos del profesor y entenderlos. Algunos incluso confiesan que, dado el carácter "aburrido" de la asignatura, prefieren turnarse para asistir a clase y ejercer de copistas de todo lo afirmado por el profesor, pasándose unos a otros los apuntes.

Como ya he puesto de manifiesto, en muchos casos estos apuntes poseen numerosas incongruencias, por lo que no resulta extraño encontrar los mismos fallos en las respuestas de los exámenes. Y todo ello a pesar de que, al igual que otros colegas, habitualmente suelo recomendar a mis alumnos que accedan directamente a las fuentes bibliográficas del programa, o a aquellas que les voy sugiriendo antes de la explicación de un tema determinado. Sin embargo, la mayoría de los estudiantes no suele prestar atención a dicha recomendación y únicamente se limita a copiar apuntes. Asimismo, el rechazo que muchos de ellos experimentan hacia la asignatura hace que no la lleven al día y la mayor parte se limita a tratar de estudiar unos contenidos que no comprende, únicamente con el fin de poder plasmarlos en el examen, olvidándolos a las pocas horas.

Los contenidos conceptuales de la asignatura, tal y como se recogen en el programa docente desde su inclusión en 2009 en el plan de estudios del Grado en Filología Hispánica, se organizan en los siguientes bloques temáticos:

1) Introducción a los modelos de explicación gramatical de la lengua española.

2) Las unidades gramaticales. 
3) El sujeto.

4) El predicado.

5) La oración. Tipología oracional.

6) De la oración al discurso.

Tales bloques temáticos decidí a la hora de elaborar el proyecto docente que podían concretarse en catorce temas, como muestro en la Figura 1:

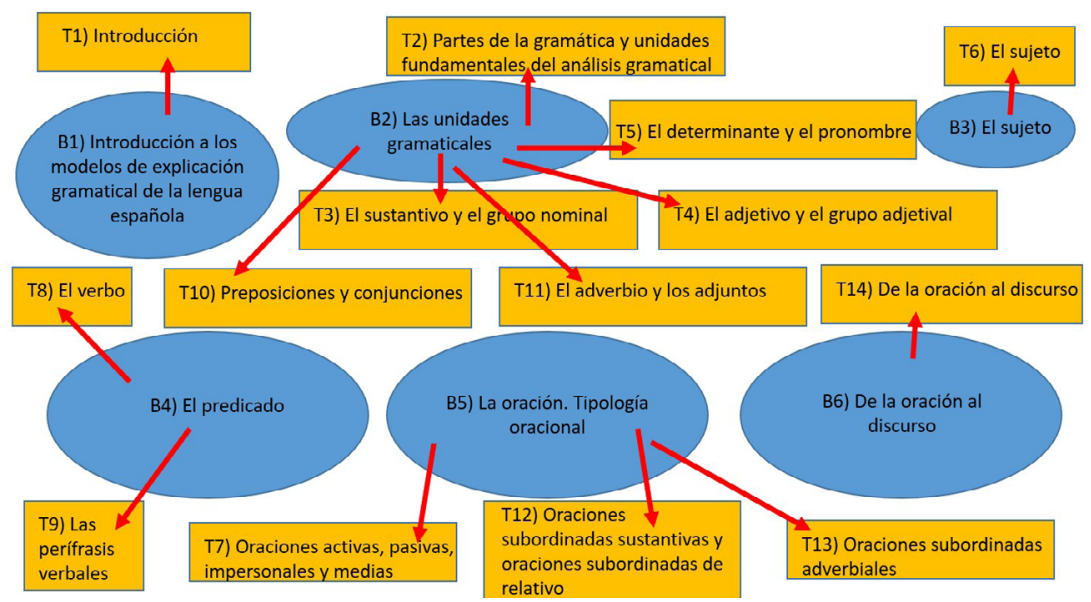

Figura 1. Programación global de la asignatura en el proyecto docente

En la Figura 2 se presentan los resultados de aprendizaje y en la Tabla 1 las competencias -básicas, generales y específicas- que se recogen en la memoria de verificación del título:

-Adquisición de un conocimiento adecuado de los problemas gramaticales del español que han suscitado el interés de los principales modelos explicativos.

-Adquisición de capacidad crítica sobre dichos modelos teóricos.

-Aplicación de los conocimientos teóricos en la resolución de los problemas prácticos planteados (análisis de textos de diversa tipología y procedencia).

Figura 2. Objetivos de aprendizaje extraídos de la memoria de verificación del título 
Tabla 1. Competencias que figuran en la memoria de verificación del título

\begin{tabular}{|c|c|c|}
\hline \multicolumn{3}{|c|}{ COMPETENCIAS } \\
\hline BÁSICAS & GENERALES & ESPECÍFICAS \\
\hline $\begin{array}{l}\text { CB1 Que los estudiantes } \\
\text { hayan demostrado poseer y } \\
\text { comprender conocimientos en } \\
\text { un área de estudio que parte } \\
\text { de la educación secundaria } \\
\text { general e incluye también } \\
\text { algunos aspectos que implican } \\
\text { conocimientos procedentes de } \\
\text { la vanguardia de su campo de } \\
\text { estudio. } \\
\text { CB2 Que los estudiantes sepan } \\
\text { aplicar sus conocimientos a } \\
\text { su trabajo o vocación de una } \\
\text { forma profesional y posean } \\
\text { las competencias que suelen } \\
\text { demostrarse por medio de } \\
\text { la elaboración y defensa de } \\
\text { argumentos y la resolución de } \\
\text { problemas dentro de su área de } \\
\text { estudio. } \\
\text { CB3 Que los estudiantes } \\
\text { tengan la capacidad de reunir } \\
\text { e interpretar datos relevantes } \\
\text { (normalmente dentro de su área } \\
\text { de estudio) para emitir juicios } \\
\text { que incluyan una reflexión sobre } \\
\text { temas relevantes de índole } \\
\text { social, científica o ética. } \\
\text { CB4 Que los estudiantes puedan } \\
\text { transmitir información, ideas, } \\
\text { problemas y soluciones a un } \\
\text { público tanto especializado como } \\
\text { no especializado. } \\
\text { CB5 Que los estudiantes } \\
\text { hayan desarrollado aquellas } \\
\text { habilidades de aprendizaje } \\
\text { necesarias para emprender } \\
\text { estudios posteriores con un alto } \\
\text { grado de autonomía. }\end{array}$ & $\begin{array}{l}\text { G01 Capacidad de aplicar } \\
\text { los conocimientos en la } \\
\text { práctica. } \\
\text { G02 Conocimientos } \\
\text { generales básicos sobre } \\
\text { su área de estudio. } \\
\text { G03 Conocimientos } \\
\text { básicos de la profesión. } \\
\text { G04 Capacidad para } \\
\text { comunicarse con } \\
\text { personas no expertas en } \\
\text { la materia. } \\
\text { G05 Habilidad para } \\
\text { trabajar de forma } \\
\text { autónoma. } \\
\text { G06 Capacidad de } \\
\text { análisis y síntesis. } \\
\text { G07 Capacidad de } \\
\text { aprender. } \\
\text { G08 Capacidad crítica y } \\
\text { autocrítica. } \\
\text { G09 Trabajo en equipo. } \\
\text { G10 Habilidades } \\
\text { personales. }\end{array}$ & $\begin{array}{l}\text { E01 Dominio instrumental } \\
\text { de la lengua española. } \\
\text { E02 Conocimiento de la } \\
\text { gramática del español. } \\
\text { E04 Capacidad para } \\
\text { comunicar y enseñar } \\
\text { los conocimientos } \\
\text { adquiridos. } \\
\text { E05 Conocimiento de las } \\
\text { técnicas y métodos del } \\
\text { análisis lingüístico. } \\
\text { E07 Capacidad para } \\
\text { analizar textos y } \\
\text { discursos literarios y } \\
\text { no literarios utilizando } \\
\text { apropiadamente las } \\
\text { técnicas de análisis. } \\
\text { E08 Capacidad para } \\
\text { realizar análisis y } \\
\text { comentarios lingüísticos. } \\
\text { E09 Conocimiento de la } \\
\text { variación lingüística de la } \\
\text { lengua española. }\end{array}$ \\
\hline
\end{tabular}


En especial consonancia con las competencias CB4, G01, G04, G05, G06, G09, G10, E04, E07 -aunque sin dejar de lado todas las demás-, diseñé el CIMA basándome en las siguientes innovaciones:

1) Estructurar la mayoría de las sesiones en clases en las que los propios estudiantes, organizados en grupos, tendrían que explicar los contenidos a sus compañeros.

2) Suministrar a los alumnos todos los contenidos que debían estudiar en cada tema. En la página web de la asignatura, los estudiantes podrían descargar la totalidad de los contenidos objeto de examen.

3) En las exposiciones no podrían utilizarse los ejemplos que figuran en la bibliografia, sino que los estudiantes tendrían que buscar sus propias muestras de lengua para ilustrar cada uno de los fenómenos gramaticales que explicaran.

4) Todos los grupos tendrían que elaborar un mapa conceptual y un power point en el que se resumieran los principales aspectos del tema que les correspondiera exponer, con el fin de que estos pudieran servir como complemento de estudio para sus compañeros.

5) Las exposiciones se irían alternando con clases prácticas que correrían a mi cargo, en las que trataría de que los alumnos se familiarizasen con los contenidos de los temas tratados en las sesiones previas, mediante ejercicios que realizaríamos en clase y en los que tendrían que analizar cuestiones gramaticales presentes en textos de distinto tipo.

6) Los estudiantes deberían entregar un porfolio en el que se incluyeran las resoluciones de las prácticas, un "diario del alumno", las rúbricas de valoración de las exposiciones de cada uno de los grupos, la rúbrica de valoración de su propio grupo y una encuesta de autoevaluación -esta cuestión la explicaré detenidamente más adelante-. 
A modo de síntesis de lo expuesto en este epígrafe, podríamos representar el modelo metodológico seguido tradicionalmente en la docencia de la asignatura Gramática española y el diseñado para este CIMA de la siguiente forma (Figura 3):
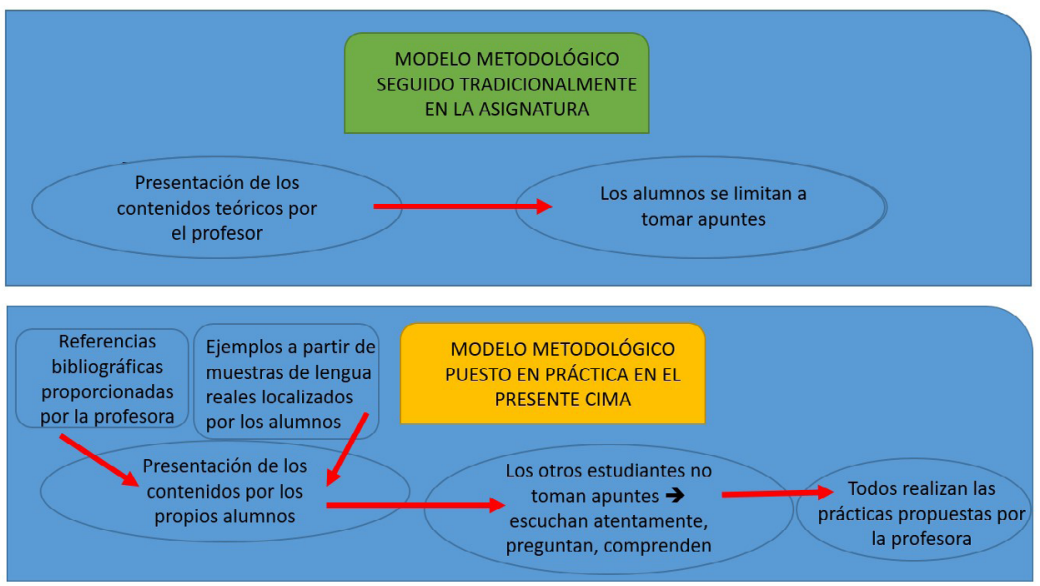

Figura 3. Esquema de los modelos metodológicos aplicados en la docencia de Gramática Española

Así, en el diseño de este CIMA pretendí que fueran los propios alumnos los que, en grupos reducidos, llevaran a cabo la exposición de los contenidos del temario. Esta organización de la docencia permitiría que los estudiantes adquirieran también contenidos actitudinales y procedimentales relacionados, por ejemplo, con la capacidad de trabajar en equipo, de elaborar una unidad didáctica, de exponer en clase los resultados de su propio análisis de textos de distinto tipo, de conocer los modelos mentales de sus compañeros y relacionarlos con los suyos, de explicar de manera sencilla nociones complejas vinculadas con los saberes principales de la disciplina, de mejorar sus competencias de expresión oral y de comprensión auditiva, etc. En cuanto a mi papel, desde el principio tuve claro que debía abandonar la posición elevada que me confería la tarima -tanto en sentido literal como metafórico-, y 
actuar como orientadora del proceso de construcción de conocimiento de mis estudiantes (De Alba y Porlán, 2017).

\section{Aplicación del CIMA}

Gramática española es una asignatura de carácter obligatorio que los alumnos que se encuentran en el cuarto año del Grado en Filología Hispánica deben cursar a lo largo del primer cuatrimestre. Actualmente, se ofertan dos grupos para esta asignatura: uno en el horario de mañana y otro en el de tarde. Las clases se imparten tres días a la semana con una duración de 80 minutos cada una. Al igual que otros años, durante el curso 2019/2020 me he encargado de la docencia del grupo de tarde, en el que se ha matriculado un total de 79 alumnos. A algunos de ellos ya les di clase hace tres años, en el primer curso del Grado en Filología Hispánica, en las asignaturas Lengua española I y Lengua española II. Esto tiene para mí dos ventajas: 1) Ya conozco algunos de sus nombres y ellos están familiarizados con mi manera de enfocar las clases, lo que me permite que el ambiente sea distendido -algo que para la puesta en práctica de este ciclo de mejora resulta fundamental-; 2) Los contenidos de Lengua española I y Lengua española II guardan una estrecha relación con los que figuran en el programa de Gramática española. Como pude comprobar al leer sus respuestas al cuestionario inicial, la mayoría de mis estudiantes no recordaba algunos de los conceptos que se abordaron de manera introductoria en dichas asignaturas, pero durante las sesiones prácticas diseñé varias actividades para tratar de "rescatar" aquellos recuerdos. Por ejemplo, mediante el análisis sintáctico del tuit que muestro en la Figura 4:

Dios@Sr_Dios.5 sept.

No puedo crear sueños para todos, por eso algunas noches no puedes dormir. Tienes que esperar a que alguien se despierte para que te toque tu turno.

Q $12 \quad \uparrow 】 66 \quad$ O 320 个

Figura 4. Tuit analizado sintácticamente durante una de las clases prácticas 
Durante las tres primeras semanas del curso me encargué de exponer los dos temas iniciales y de organizar el trabajo en los distintos grupos, bajo mi supervisión directa. Además de realizar la actividad de diseñar la estructura de una gramática redactada por ellos mismos -tal y como he explicado en el epígrafe introductorio-, tuvieron que leer detenidamente un fragmento del prólogo de la Nueva gramática de la lengua española (2019) y elaborar en grupos, fuera del aula, para que pudieran trabajar con mayor comodidad- una línea temporal en la que aparecieran reflejados los distintos acontecimientos relacionados con la elaboración de las diversas ediciones de la gramática académica. Posteriormente, presentaron sus propuestas a los compañeros, que votaron cuál era la que les resultaba más útil para el estudio. La opción elegida es la que presento en el Figura 5 -esta es la que ha quedado recogida en el power point del tema 1-:

GRUPO 3

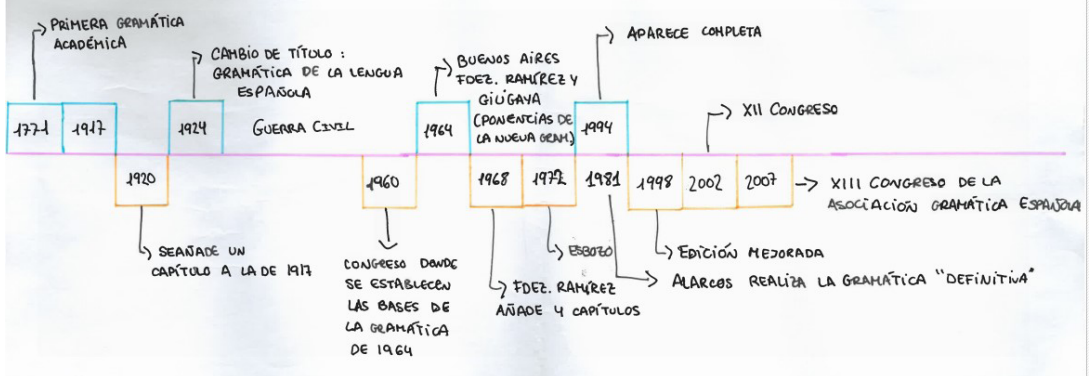

Figura 5. Línea temporal presentada por uno de los grupos

Para la exposición del resto de los contenidos teóricos de los temas 1 y 2 me fui sirviendo de preguntas focalizadas en distintos problemas. A continuación, presento algunas de ellas, a modo de muestra: “¿Creéis que han cambiado mucho los estudios gramaticales desde la anterior edición de la gramática de la Real Academia Española (1931) hasta la actual?"; "Si hubierais tenido que elaborar 
vosotros la Nueva gramática de la lengua española (NGLE) ¿qué hubierais hecho, romper con toda la tradición gramatical anterior y recoger todas las innovaciones que han tenido lugar desde 1931 hasta la actualidad o no?"; "¿En vuestra gramática presentaríais el español de un determinado país como modelo panhispánico de lengua, o trataríais de realizar una descripción detallada de las distintas variedades del español americano y del europeo? ¿qué creéis que se hizo en la NGLE?”; “En vuestra gramática realizaríais una descripción de todos los usos, incluso de los antinormativos, o solo de los acordes con la norma?"; “A qué tipos de variación se hace referencia en la NGLE?"; “¿Cuáles creéis que son las fuentes de las que se extraen los ejemplos en la NGLE?"; “Qué es la gramática? ¿qué subdisciplinas abarca?"; “El estudio del significado es ajeno a la gramática?"; “¿A la hora de elaborar una gramática deben tenerse en cuenta también aspectos pragmáticos?”; “Qué tipos de gramáticas conoces?”, etc. A raíz de esas preguntas, aproveché para mostrarles cómo se habían conseguido solventar dichos problemas en la tradición gramatical y cómo había sido la génesis de la NGLE. Además, les hablé de los distintos tipos de gramáticas existentes, enseñándoles también a valorar los datos gramaticales.

El resto de las sesiones -excepto aquellas de carácter práctico- se desarrollaron en forma de clases invertidas. Sobre dicha metodología docente versan, por ejemplo, los trabajos de Bergmann y Sams (2012), Tourón y Santiago (2015) o Fernández Gámez y Guerra (2016), entre otros. Esta me parece muy adecuada para los alumnos que están concluyendo el Grado en Filología Hispánica pues, en general, poseen gran temor a hablar en público, su competencia de expresión oral no es excesivamente elevada y se encuentran con graves problemas a la hora de tratar de comprender por sí mismos, sintetizar y explicar aspectos clave de la bibliografia especializada sobre aspectos gramaticales. Pienso que esto puede suponer un grave lastre para su 
incorporación al mercado laboral. Por ejemplo, como docentes en la enseñanza secundaria o en la universidad, o como profesores de español para extranjeros -estas son las salidas profesionales a las que aspira la mayoría de mis estudiantes-.

Mi propósito era que, mediante esta metodología los alumnos desarrollaran las siguientes actividades: a) Leer detenidamente las "notas bibliográficas" del tema que tuvieran que exponer; b) tratar de comprender todo lo que ahí se mencionaba; c) preguntarme las dudas; d) organizar los contenidos de las "notas bibliográficas" en epígrafes; e) buscar ejemplos reales para ilustrar cada fenómeno expuesto en la teoría; f) elaborar un mapa conceptual y un power point que pudiera servir de ayuda al resto de los compañeros para estudiar el tema; g) preparar y ensayar su exposición con el fin de lograr ajustarse al tiempo estipulado; h) explicar su tema y tratar de responder a las preguntas de sus compañeros -obviamente, para realizar tales tareas pudieron contar con mi ayuda a lo largo de todo el proceso-.

En la anterior asignatura en la que puse en práctica un ciclo de mejora completo, Análisis lingüístico de textos del español actual (Mancera, 2018), utilicé un sistema similar: para cada tema, yo subía a la página web dos o tres artículos y los miembros del grupo encargado de explicarlo al resto de la clase tenían que leerlos por completo y realizar una sintesis de dichos artículos, descartando aquellos aspectos que no consideraban fundamentales. Este sistema -a pesar de que ha proporcionado muy buenos resultados en la asignatura mencionada- no me parece conveniente para Gramática española, donde los contenidos son más complejos. Así, soy consciente de que no puedo pedir a un alumno de grado que lea un capítulo de ochenta páginas de la Nueva gramática de la lengua española, elaborada por la Real Academia Española, y que seleccione cuáles son los aspectos fundamentales que debe conocer, 
o que lea un artículo de investigación sobre un determinado aspecto gramatical y que sea capaz de comprenderlo en su totalidad, así como de sintetizarlo. Por estas razones, me encargué de redactar las "notas bibliográficas para la elaboración de cada tema" -así las denomino-, algo que me llevó muchas horas de trabajo. Para ello, fui seleccionando y copiando fragmentos de las monografias y artículos que consideré fundamentales para que los alumnos estudiaran los contenidos del programa. Todas estas "notas bibliográficas" tenían una extensión similar, con el fin de que los contenidos que ahí se presentaban pudieran exponerse y explicarse a un ritmo no excesivamente rápido, durante dos sesiones por tema.

Desde mi punto de vista, parte del trabajo que un docente tiene que realizar habitualmente consiste en leer una ingente cantidad de bibliografia especializada e ir organizando cada tema en distintos epígrafes. A veces, es necesario contraponer la perspectiva de distintos autores en un mismo epígrafe. Dado que la mayor parte de los alumnos del Grado de Filología Hispánica van a ser docentes, me parece importante que aprendan a organizar ellos mismos los contenidos del tema que tengan que explicar. Como ya he puesto de manifiesto anteriormente, soy consciente de que no puedo pedir a mis alumnos que en una asignatura tan compleja seleccionen la bibliografía que debe utilizarse para la explicación de un aspecto gramatical, por eso les suministré los contenidos que tenían que estudiar, pero limitándome a presentar los fragmentos de las investigaciones de distintos autores copiadas unas a continuación de las otras, con el fin de que fueran los propios estudiantes los que organizasen tales contenidos estructurándolos en epígrafes.

Otra de las competencias que me parecía importante que los alumnos del Grado en Filología Hispánica adquirieran, para poder desempeñar su actividad laboral como docentes más satisfactoriamente, consistía en ser capaces 
de localizar los ejemplos adecuados para ilustrar un determinado fenómeno gramatical. Con el fin de ayudarles a desarrollar esta destreza, decidí introducir otra innovación en mi ciclo de mejora: en la explicación de un tema, los estudiantes encargados de hacerlo no podían limitarse a utilizar los ejemplos que figuraban en la bibliografia que yo les había proporcionado, sino que tenían que buscar sus propios ejemplos para ilustrar cada uno de los aspectos teóricos mencionados. Para ello les enseñé a consultar la base de datos CREA (http://corpus.rae.es/creanet.html) y diversas hemerotecas digitales en español.

\section{Evaluación del CIMA puesto en práctica}

En cuanto a la evaluación del aprendizaje, para explorar las hipótesis previas de los estudiantes en relación con los contenidos presentados, se elaboró un cuestionario inicial compuesto por diez preguntas sobre los seis primeros temas, y al que los estudiantes tuvieron que responder, de nuevo, a mediados de noviembre de 2019 -este es el plazo fijado por el Instituto de Ciencias de la Educación de la Universidad de Sevilla (ICE) para la entrega del presente capítulo-. Al contestar el cuestionario, pedí a los alumnos que utilizaran un seudónimo, el cual tendrían que volver a emplear al responder a las preguntas de ese mismo cuestionario por segunda vez. Esto me permitió advertir que existía una ligera diferencia entre el número de alumnos que respondieron al cuestionario en ambas ocasiones, por esta razón he decidido cuantificar exclusivamente aquellos cuestionarios para los que contaba con una versión inicial y una versión final, con el objeto de poder establecer una comparación. Es decir, en el cómputo voy a tener en cuenta solamente 42 alumnos, que fueron los que contestaron a las dos versiones del cuestionario. El hecho de poder utilizar un seudónimo sorprendió a los alumnos e introdujo un aspecto lúdico en la clase, 
al permitirles adoptar identidades de lo más variopintas: Perifrástico, El caballero de la triste figura, Alumna estresada, La vaga del pueblo - paradójicamente, este cuestionario presentaba la mayoría de las preguntas resueltas, luego cabe pensar que su autor o autora no se había identificado plenamente con el rol adoptado-, etc.

El análisis de las respuestas de los alumnos al cuestionario me ha permitido observar, en primer lugar, cómo el número de preguntas que los estudiantes habían dejado sin contestación en la primera clase era sorprendentemente elevado (13,2\%). Esta circunstancia me ha llevado a considerar un modelo A basado en las respuestas en blanco que, lógicamente, no conlleva ningún esfuerzo por parte del alumno. Pienso que esto puede deberse a que ellos desconocían las respuestas a las preguntas o es posible incluso que por pereza hayan preferido no contestarlas. Hay que tener en cuenta que la mayor parte de estas preguntas que dejaban sin respuesta conllevaban la realización de un análisis de ciertos aspectos gramaticales y que antes de poner en práctica este CIMA, muchos alumnos adoptaban una actitud escasamente participativa en clase. Por fortuna, tras el análisis de la escalera final advertí cómo el número de respuestas en blanco parecía haber descendido a la mitad. Así, el modelo A ha pasado a tener una representatividad del 6,8\%.

Por otra parte, la observación de las respuestas de los alumnos, me permitió darme cuenta de que en muchas ocasiones estos proporcionaban lo que he denominado unas "respuestas ejemplificadoras sin reflexión lingüística". En ellas, los alumnos parecían fundamentar sus contestaciones exclusivamente en el conocimiento que les proporcionaba su competencia como hablantes nativos de español. Por ejemplo, al corregir los errores gramaticales de la siguiente pregunta, pero sin argumentar en qué se basaba su contestación (Figura 6): 
10)Señala los errores que pueda haber en el uso de los determinantes en los enunciados siguientes. Corrígelos y argumenta por qué:
a. Ese arca es más anchôue este.
a
b. Hoy tengo el mismo agua que ayer.
la cuisua
c. Nunca digas "de esłe agua no beberé".
esta
d. Hemos pasado muche hambre.
a
e. En todo estrárea se van a construir pisos.

\section{a}

Figura 6. Extracto de la pregunta 10 del cuestionario

Tal tipo de respuestas las he identificado bajo el marbete de modelo B. Un modelo que contaba con una representación inicial del 68,7\% -la mayoría de los alumnos hacía uso de este tipo de respuestas-, pero que en la escalera final pasó a representar el 32,9\%.

Dentro del modelo $\mathrm{C}$ he incluido aquellas respuestas que conllevaban lo que he identificado como "respuestas basadas en conocimientos gramaticales básicos" que, por ejemplo, les llevaba a definir una aposición como una "palabra que funciona como explicación de otra anterior", o como "un nombre que acompaña a otro nombre". La representatividad de este tipo de modelo ha aumentado, al pasar de un 15,2\% a un 24,5\%, mostrando el esfuerzo de algunos estudiantes por proporcionar una respuesta basada en contenidos gramaticales, más allá de la mera corrección de una construcción agramatical, como se hacía en el modelo $\mathrm{B}$.

Por último, el modelo D -el de mayor complejidad- lo he identificado como "respuestas basadas en conocimientos gramaticales avanzados". Con ellas, los estudiantes 
demostraban que sabían distinguir entre los conceptos de agramaticalidad e incorrección lingüística, y entre la noción de complemento argumental y la de adjunto, que eran capaces de identificar los grupos nominales escuetos o definir las aposiciones como "secuencias en las que un sustantivo o grupo nominal incide sobre otro y da como resultado una expresión sintáctica", y poner ejemplos que ilustraran que dominaban el metalenguaje propio de esta área de la lingüística. La comparación de las dos escaleras -la inicial y la final- me ha permitido advertir que son muchos los alumnos que parecen haber dado un gran paso en su aprendizaje, ya que la representatividad de este modelo se ha incrementado considerablemente -del 2,9\% al $35,8 \%-$. En las Figura 7 he representado en forma de escalera los datos aquí expuestos:

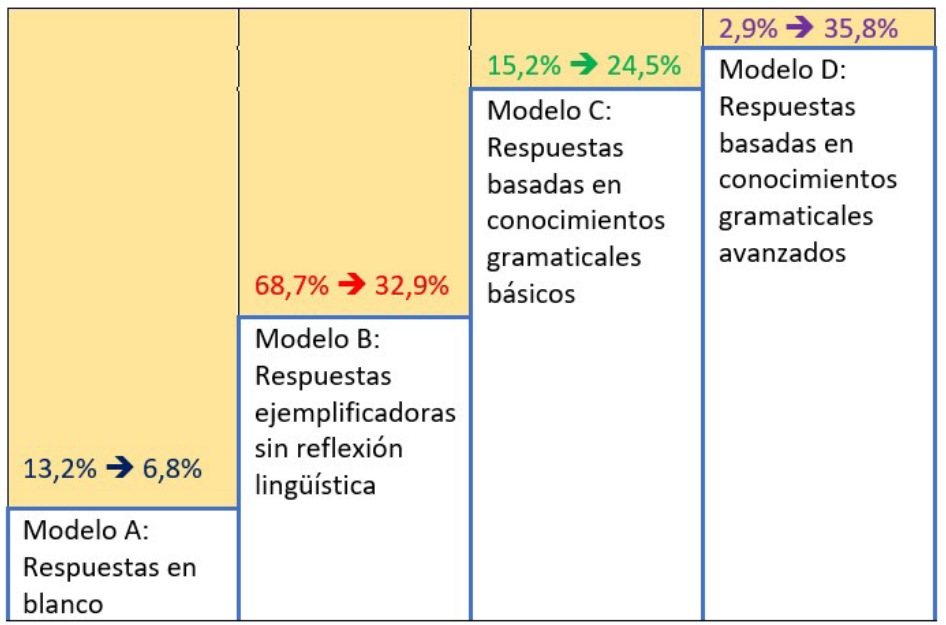

Figura 7. Escaleras basadas en el análisis de las respuestas al cuestionario inicial-final

Tal y como se especifica en el proyecto docente, el sistema de evaluación de esta asignatura se fundamenta en los siguientes aspectos:

1) Examen parcial optativo: $20 \%$ de la calificación global. 
2) Examen final: $40 \%$ o $60 \%$ de la calificación global en el caso de que el alumno no hubiera realizado el examen parcial o de que la calificación de este fuera inferior a la del final, ya que el parcial no se tendría en cuenta-.

3) Exposición grupal de una de las lecciones del temario: $20 \%$ de la calificación final.

4) Porfolio: $10 \%$ de la calificación global.

5) Asistencia a clase: $10 \%$ de la calificación global.

Respecto al examen parcial, aunque solo se presentaron 32 alumnos, los resultados fueron bastante buenos y algunos de ellos me comentaron que les había servido para tratar de llevar la asignatura al día y, al mismo tiempo, para hacerse una idea de cómo iba a ser el examen final. Por lo tanto, creo que la idea de plantear el examen parcial en esta asignatura ha sido algo positivo, aunque para mí haya supuesto un incremento en la carga de trabajo.

Por otra parte, las exposiciones grupales de las lecciones del temario tenían que ser calificadas por los propios compañeros mediante rúbricas donde se evaluara a cada grupo. Estas debían incluirse en el porfolio, junto a los siguientes contenidos:

1) La resolución de las actividades prácticas propuestas por la profesora a lo largo de las clases impartidas por ella.

2) Diario del alumno: resumen valorativo sobre las distintas sesiones -también se podía estructurar por temas-. Extensión máxima: 250 palabras para el resumen de cada sesión o 500 palabras para el resumen de un tema -este debía redactarse adecuadamente, es decir, el alumno no podía limitarse a incluir una somera relación de contenidos con guiones o a copiar párrafos de la bibliografia-.

3) Rúbrica de valoración de cada uno de los grupos. 
4) Rúbrica de valoración de la exposición del propio grupo.

5) Autoevaluación del curso y del trabajo personal realizado a lo largo de todo el cuatrimestre.

Con este tipo de actividades mi propósito era, por una parte, lograr que todos los alumnos se enfrentaran a la tarea de realizar las prácticas -no solo los que hubieran acudido a clase esos días-y, por otro, ayudar a desarrollar su espíritu crítico y su capacidad argumentativa, tanto en el diario del alumno como en las rúbricas y en la autoevaluación, al verse en la necesidad de razonar la propia calificación y la otorgada a otros grupos. Al mismo tiempo, tales valoraciones podían servirme como instrumento de mejora de cara a la impartición de la asignatura en el próximo curso. Dado que en el momento de redactar estas páginas -noviembre de 2019-, quedan varias semanas para que concluya el curso y, lógicamente, los alumnos no han podido enviarme aún el porfolio -pueden hacerlo hasta la fecha del examen final, fijada para la primera o la segunda convocatoria-, no poseo evidencias para poder analizar tales aspectos.

Sin embargo, sí cuento con algunos datos sobre cómo han acogido los alumnos las exposiciones de sus compañeros. Y es que durante este CIMA me he decidido a utilizar por primera vez una aplicación que Charo Martínez Navarro y Juan Pablo Mora me enseñaron a manejar en su curso del ICE sobre Cómo implicar a los estudiantes a través de nuevas herramientas tecnológicas. Se trata de Padlet, una plataforma en la que los alumnos pudieron ir escribiendo sus valoraciones acerca de las exposiciones de sus compañeros, insertando sus comentarios en los apartados \#AsíSí, \#Asíno, \#Ysi. En la Figura 8 muestro una captura de pantalla del muro de Gramática española: 


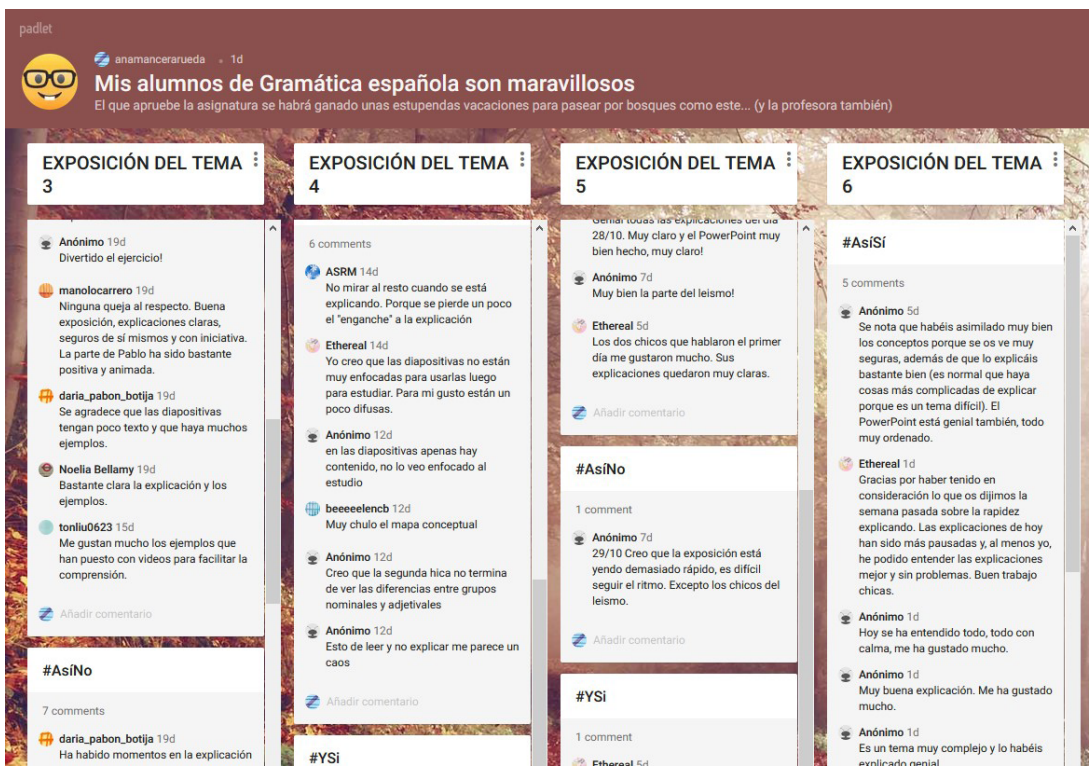

Figura 8. Captura de pantalla del muro de la asignatura en la aplicación Padlet

Debo reconocer que me ha sorprendido mucho la manera natural de la que los estudiantes han integrado esta aplicación en el aula. Inicialmente, pensé que ellos serían reticentes a utilizarla, pero no ha sido así. A lo largo de todas las clases iban tecleando sus comentarios -bien por medio de su teléfono móvil o de su ordenador-, ya fuera de forma anónima o proporcionando sus datos personales, y estos eran tenidos en cuenta por los miembros de un determinado grupo, a modo de propuestas de mejora, entre su primera y su segunda sesión. De hecho, en algunos casos pude apreciar cambios muy positivos en la forma de abordar las explicaciones entre una y otra sesión, motivados por las sugerencias proporcionadas a través de Padlet. Además, tal y como aconsejé el primer día de clase, dichas sugerencias se redactaron siempre de manera constructiva, para no alterar el clima positivo del aula. Reproduzco a continuación, a modo de muestra, algunos de estos comentarios: 
\#Asísí

- Poco texto por diapositiva está bien para que podamos asimilar más fácilmente.

- Muy buena la explicación de Margarita al principio. Me gustan las imágenes!

- La información está muy bien organizada. Hace fácil seguir el hilo!

\#Asíno

- Creo que la exposición está yendo demasiado rápido, es dificil seguir el hilo. Excepto los chicos del leísmo!

- Algunas veces han explicado un poco rápido pero en general ha sido una explicación exitosa.

\#Ysi

- Hubiese agradecido que hubiese más cosas en negrita o con colores varios para que visualmente sea más fácil entender la exposición.

En cuanto a mi propia valoración acerca de las exposiciones, debo reconocer que me siento bastante satisfecha. Por lo tanto, pienso incorporar este modelo metodológico a mi práctica docente habitual en otras asignaturas, poniendo el foco, no tanto en el "saber", como en el "saber hacer". En este caso, el saber transmitir los principales problemas gramaticales y analizar críticamente cualquier tipo de texto. Mediante las clases impartidas por los propios alumnos, intenté favorecer un "aprendizaje autodirigido" (Marcelo, 2010), potenciando la autonomía y la responsabilidad de los estudiantes, así como el trabajo colaborativo y el sentido crítico. Esos han sido los principios didácticos que han guiado este CIMA y son los que deseo seguir manteniendo.

Me siento también muy satisfecha por el hecho de que todos los grupos respetaran los plazos de entrega de su power point y su mapa conceptual, que fueron 
supervisados por mí. Además, les animé a ensayar sus presentaciones con anterioridad y a trabajar en equipo, haciendo que la totalidad de los miembros de un grupo se encontraran presentes en el aula durante las dos sesiones correspondientes a su tema. No se trataba de que cada integrante de un equipo se limitara a conocer únicamente los contenidos que le tocaba presentar, sino de que existiera una verdadera colaboración entre todos, de manera que, si alguno no sabía responder a las preguntas formuladas por los presentes, los demás pudieran ayudarle. Y así ocurrió en algunos casos. Asimismo, todos los grupos supieron ajustarse perfectamente al tiempo de exposición que les correspondía. En referencia a la organización de las "notas bibliográficas" en distintos epígrafes, pienso que esto ha constituido un gran acierto, que ha contribuido a desarrollar su mentalidad crítica, llevándoles a identificar los puntos en común entre las obras de los autores mencionados o a contrastar distintas teorías. Por lo que respecta a la búsqueda de ejemplos, varios grupos superaron con creces mis expectativas, puesto que presentaron muestras de lengua extraídas de películas como las que conforman la saga de Harry Potter o series de televisión como Juego de Tronos o Aquí no hay quien viva, lo que demostraba que habían invertido una gran cantidad de tiempo en tratar de localizar en estos productos audiovisuales los fenómenos gramaticales expuestos en la teoría, esforzándose al mismo tiempo por lograr que la clase resultara amena para sus compañeros, algo que también lograron introduciendo la teoría con preguntas que lograron captar su interés -“Pregunta de oposiciones: ¿qué son las aposiciones?"; "¿Navidad es un nombre común o un nombre propio? ¿y si lleva artículo?"; “¿Cuántos tipos de sustantivos conocéis?"; "¿Podéis completar los títulos de las siguientes películas con su adjetivo correspondiente: Instinto ..., La naranja ...?"-. Además, muchos utilizaron la herramienta Kahoot a modo de repaso del tema y organizaron concursos -por ejemplo, a partir del popular juego de mesa Dixit- en los que la participación era incentivada 
con gominolas, frutos secos, donuts o, incluso, raciones de croquetas. En este sentido, podría decirse irónicamente que la profesora fue la única que dio "clase con la boca cerrada" (Finkel, 2000). 


\section{Referencias bibliográficas}

Bergmann, J. y Sams, A. (2012). Flip your Classroom: Reach Every Student in Every Class Every day. Washington, DC: ISTE y Alexandria, VA: ASCD.

De Alba, N. y Porlán, R. (2017). La metodología de enseñanza. En Porlán Ariza (Coord.) Enseñanza universitaria. Cómo mejorarla (pp. 37-53). Madrid: Morata.

Fernández Gámez, D. y Guerra, M. D. (2016). Aprendizaje inverso en formación profesional: opiniones de los estudiantes. International Journal of Technology and Educational Innovation, 2(1), 29-37.

Finkel, D. (2000). Teaching with Your Mouth Shut. Porstmouth: NH. Heinemann Boynton/Cook. (Trad. cast.: Dar clase con la boca cerrada. Valencia: Publicaciones de la Universidad de Valencia, 2008).

Mancera, A. (2016). Enseñar Pragmática mediante la elaboración y el análisis de textos: una experiencia docente en el Grado en Filología Hispánica. En Porlán Ariza y Navarro Medina (Coords.) III Jornadas de Docencia Universitaria. Universidad de Sevilla (30 de junio y 1 de julio de 2016) (pp. 973-985). Sevilla: Universidad de Sevilla.

Mancera, A. (2018). Una experiencia de clase invertida en el Grado en Filología Hispánica. Jornadas de Formación e Innovación Docente del Profesorado, 1, 390-410.

Marcelo, C. (2010). Autoformación para el siglo XXI. En Gairín Sallan (Coord.) Nuevas estrategias formativas por las organizaciones (pp. 141-170). Madrid: Wolters Kluwer.

Pons, L. (2018). En clase de lengua. El País, 9 de octubre de 2008. Recuperado de

https://elpais.com/elpais/2018/10/04/opinion/1538669568 413238.html

Porlán, R. (2016). Programa integrado de Formación e Innovación docente centrada en el aula (FIDA): un objetivo estratégico para el III Plan Propio de Docencia. Sevilla: Universidad de Sevilla.

Real Academia Española/ Asociación de Academias de la Lengua Española (2009). Nueva gramática de la lengua española. Madrid: Espasa Libros.

Tourón, J. y Santiago, R. (2015). El modelo Flipped Learning y el desarrollo del talento en la escuela. Revista de Educación, 368, 196-231. 